\title{
Plastid terminal oxidases in Chlamydomonas: connections with astaxanthin and bio-hydrogen production
}

Hui Li

Shenzhen university

Chao Zheng

Shenzhen University

Ming Xiao

Shenzhen University

Qin Huan

Shenzhen University

Jun Chen

Shenzhen University

Mingjie Dong

Shenzhen University

Wenlong Hao

Shenzhen University

Zhangli Hu

Shenzhen University

Anping Lei

Shenzhen University

Jiangxin Wang ( $\square$ jxwang@szu.edu.cn )

https://orcid.org/0000-0002-9917-067X

\section{Research}

Keywords: plastid terminal oxidase, microalgae, biofuels, bio-hyodrgen, RNAi

Posted Date: April 7th, 2020

DOI: https://doi.org/10.21203/rs.3.rs-21273/v1

License: (c) (1) This work is licensed under a Creative Commons Attribution 4.0 International License.

Read Full License 


\section{Abstract}

Background: as a plasto quinol oxidase involved in plastoquinol oxidation in higher plants and microalgae, the plastid terminal oxidase (PTOX) was first recognized in the tomato mutant GHOST (GH) and Arabidopsis mutant IMMUTANS (IM). Genome sequence analysis revealed that duplication of the PTOX gene occurs in certain eukaryotic microalgae, but not in cyanobacteria and most higher plants. PTOX may also be involved in carotenoid synthesis and play a critical protective role against stress, such as high light, heat shock and hyperosmosis. However, the connections of PTOX with astaxanthin and biohydrogen production and their functional relationship between two PTOX genes in the model green microalga Chlamydomonas is unknown.

Results: we successfully knocked down two ptoxs through RNAi in Chlamydomonas, respectively. We demonstrated that expression levels of both PTOXs were increased under stress conditions, and interestingly when one PTOX was silenced the other's transcriptional level was significantly raised.

Conclusions: this shows a complementary relationship under high light condition. In addition, the astaxanthin accumulation level was up-regulated in silenced ptox 2 strain, compared to the wide type strain. What's more, significantly increased hydrogen production was observed in silenced ptox 1 strain. In conclusion, PTOXs in Chlamydomonas are connected with not only astaxanthin accumulation but also hydrogen production, and their knock-down strains provide new insights in manipulating microalgae for high light stress tolerant strains, carotenoid production and even biofuels.

\section{Background}

Oxygenic photosynthesis depends on a highly conserved electron transport system, which must be particularly dynamic in its response to environmental and physiological changes. This is necessary to avoid an excess of excitation energy and subsequent oxidative damage. Apart from cyclic electron flow around photosystem II (PSII) and photosystem I (PSI), several alternative electron transport pathways exist, including a plastoquinol terminal oxidase (PTOX) that mediates electron flow from plastoquinol to $\mathrm{O}_{2}$.

PTOX is a chloroplast oxidoreductase involved in carotenoid biosynthesis, chlororespiration, and responding to environmental stresses $[1,2]$. PTOX has been found to be a stress-related protein through much research and can protect plants from various environmental stresses, especially high light (HL) stress [3]. Recent studies have revealed that IM (PTOX lost mutant) plays a more global role in chlororespiration and also functions as a light stress protein [4-7]. In tomatoes, a second role of the PTOX, as a requirement for efficient carotenoid desaturation in some organs at developmental stages was reported [8]. The physiological roles of PTOX in plant stress responses were extensively studied [9], such as its involvement in the adaptation of Brassica plants to heat and HL [10], tolerance to drought [11], HL stress in cold-acclimated Arabidopsis thaliana [12], chilling stress in the tropical species Spathiphyllum wallisii [13], heat stress in Tobacco [14], and salinity stress [15]. What's more, PTOX also 
plays important roles in SA-mediated resistance to water stress during soybean seedling [16]; It was also proposed to have the potential to act as a safety valve for the excess excitation energy in the alpine plant species Ranunculus glacialis caused by rapid light and temperature changes $[17,18]$. In microalgae, by an enhanced activity of PTOX cells could decrease PSII excitation pressure in a gun4 mutant of Chlamydomonas reinhardtii [19] and PTOX responds to different environmental stress in Haematococcus pluvialis [20].

Carotenoids are accumulated at some development stage and in environmental stimuli in microalgae and higher plants, which is an acclimation strategy of the cells and helps to protect against photo-oxidative stress since carotenoids act as antioxidants [21]. The possible involvement of PTOX in the pathway of carotenoid biosynthesis [22] and chlororespiration [23] were summarized. Data show that PTOX also participates in carotenoid desaturation via a complex regulatory pattern [24] in addition to its role during early chloroplast development $[25,26]$. The $\mathrm{GH}$ terminal oxidase regulates developmental programming in tomato fruit [27] and fruit ripening [28]. Additionally, an increasing pattern of PTOX transcripts leads to carotenoid accumulation during flower bud development in Liriodendron tulipifera [29]. In another green microalga, Haematococcus pluvialis, comparative lipidomic and transcriptomic analyses reveal a concerted action of multiple defensive systems against HL stress [30] and PTOX, together with PSI cyclic electron transport, defensive enzyme and the accumulated astaxanthin, can protect microalgal cells against photoinhibition [31].

Genome sequence analysis revealed that duplication of PTOX gene occurs in certain eukaryotic algae, but not in cyanobacteria and most higher plants. In previous study in Haematococcus, two ptoxs showed differential expression patterns under different stresses [20]. The differential expression of two ptoxs in Chlamydomonas was previously reported under phosphate depletion [32]. The knockout mutant of PTOX2 in Chlamydomonas shows lower fitness than wild type when grown under phototrophic conditions [33], and another PTOX2 mutant of $C$. reinhardtii results in almost complete reduction of the plastoquinone pool in light [34]. But no further research shows the interaction between these two PTOX genes.

Hydrogen production in green mciroalgae requires electrons from the photosynthetic electron transfer chain to reduce $\mathrm{H}^{+}$into $\mathrm{H}_{2}$, especially in Chlamydomonas [35]. Since PTOX involves in chlororespiration, it is reasonable to propose that PTOX may also be one of the manipulation targets to improve the hydrogen production in microalgae.

In this study, RNA interference technique was employed to knock down two PTOXs of Chlamydomoans individually, investigating the functional relationships of these two PTOXs under stress condition. Also, the potential correlation of PTOX with astaxanthin and hydrogen bio-production was also studied.

\section{Results}

\section{PTOX knockdown and growth curves}


Firstly, we verified the successful construction of RNAi vectors using restriction digestions and DNA sequencing (Fig. S1, S2). Then more than 20 positive transformants derived from each ptox vector were screened based on their gene expressions under high light by qRT-PCR. The RNAi backbone pH124 is a high light induced vector, thus we measured expression levels of different genes under both normal and $\mathrm{HL}$ conditions. We selected the strains with the lowest ptox mRNA levels under $\mathrm{HL}, 4-6$ ( $10 \%$ of control only) and $5-3(\sim 29 \%)$, as PTOX1-RNAi (PTOX1i) and PTOX2-RNAi (PTOX2i) inferred strains for further investigations, respectively (Fig. S3).

Then we are interested in the potential correlation of PTOX knockdown and cell growth. Overall, HL inhibits cell growth in all 3 strains, PTOX1i, PTOX2i and CC849. However, RNAi mutants showed significant cell growth inhibition under $\mathrm{HL}$, while there was some insignificant difference compared with control under normal culture condition. Under normal culture condition, PTOX1i cells grow slowly after day 3 at the log phase but reached similar cell density to the control and PTOX2i showed higher cell density after 5 days culture till the platform stage, but not statistically significant (Fig. 1).

With HL treatment, cell growth in all strains were significantly inhibited, more than $30 \%$ and $56-60 \%$ inhibition in C. reinhardtii CC849 PTOX1i and PTOX2i compared to those under normal culture condition at the day 8. Though no obvious difference was observed between two mutants under $\mathrm{HL}$, the RNA interferences of both ptoxs in $C$. reinhardtii significantly inhibited cell growth compared to the $C$. reinhardtii CC849. This indicates that PTOXs may play an important role in cell growth and high light stress response.

\section{ptox 1 and ptox2 differential expression in normal and HL conditions}

Significantly different responses to cell growth were detected in two ptox genes in Chlamydomonas. Under normal condition, ptox 2 expression showed no change compared with the CC849, while ptox 1 mRNA level was being increased continuously and reached the highest level at $72 \mathrm{~h}$, about 20 fold changes compared to that of $0 \mathrm{~h}$ in both $\mathrm{C}$. reinhardtii CC849 and PTOX1i strain. When cells entered the log phase, ptox 1 started to down-regulated and reached relatively low level at $120 \mathrm{~h}$. In such normal culture condition, similar patterns were observed in both WT and PTOX1i. Combining with the cell growth curves, ptox 1 expression pattern showed highly correlation, with increasing levels at log phase and reached the maximum level at day 3 (Fig. 2a).

Interestingly, different ptox gene expression patterns were counted in different strains under HL stress (Fig. 2b, c). In this condition, the ptox 1 mRNA levels were up-regulated with more than 2 fold changes in the control cells, and its up-regulation was significantly inhibited in RNAi mutant PTOX1i (Fig. 2b). In contrast, ptox 1 was significantly increased in the PTOX2i mutant cells, with the highest up-regulation (-10 fold) observed at $48 \mathrm{~h}$ of $\mathrm{HL}$ treatment.

Gene ptox 2 seems to sensitively respond to HL in Chlamydomonas, with nearly a maximum 20 fold increases at $48 \mathrm{~h}$ in the control CC849 cells, and a similar high level at $96 \mathrm{~h}$ was also detected in PTOX1i 
mutant cells. The RNAi inhibition of ptox 2 was found after $12 \mathrm{~h}$ and relatively low expression level were recorded after $48 \mathrm{~h}$ till $120 \mathrm{~h}$ (Fig. 2c).

The interaction of ptox 1 and ptox 2 was also noted at the transcriptional level. Based on qRT-PCR analyses, the inhibition of ptox 2 in PTOX2i under $\mathrm{HL}$ resulted in the significant increase of ptox 1 with the maximum expression peak at $48 \mathrm{~h}$ with $\sim 10$ fold increase in PTOX2i compared with $\sim 1.5$ fold in PTOX1i; moreover, inhibition of ptox 1 caused a delayed up-regulation of ptox 2 in PTOX1i with a peak at $94 \mathrm{~h}$ instead of $48 \mathrm{~h}$ in CC849.

\section{Astaxanthin production}

In this study, under normal culture condition, astaxanthin was not obviously produced even after $120 \mathrm{~h}$ growth in all three strains. While there was no significant astaxanthin induction in CC849 and PTOX1i strain, interfered ptox2 in PTOX2i resulted in a significant increase of astaxanthin accumulation $(p<$ 0.05).

HL effectively induces astaxanthin accumulation in all strains, compared to normal culture condition (Fig. 3). After $120 \mathrm{~h} \mathrm{HL}$ treatment, astaxanthin in CC849, PTOX1i and PTOX2i mutants were increased 4.2, 4.8 and 5.4 fold than that of $0 \mathrm{~h} \mathrm{HL}$, respectively. RNAi mutants showed higher levels of astaxanthin accumulation compared to the control under HL, PTOX2i and PTOX1 $\mathrm{i}$ cells contained almost 2.3 and 1.3 fold astaxanthin after $120 \mathrm{~h}$ compared to $0 \mathrm{~h} \mathrm{HL}$ treatment, accordingly. Similarly, only PTOX2i mutant cells showed obviously higher astaxanthin accumulation than CC849 and PTOX1i cells after $120 \mathrm{~h} \mathrm{HL}$ treatment.

\section{Bio-hydrogen production}

To explore the consequence of PTOX inhibitions under HL, the bio-proudction of hydrogen was determined in all three strains. Microalgae cells were cultured in normal condition until exponential phase and then treated with $\mathrm{HL}$, and then the hydrogen production was determined, using gas chromatography

to measure the content of gas in the headspace of WT and RNAi strains. It was observed the significantly increased hydrogen production in PTOX1i mutant under HL treatment, whereas there was slightly but not significantly increase in the other RNA interfered mutant PTOX1i compared to the control $C$. reinhardtii CC849 (Fig. 4), indicating HL inhibition of PTOX1 and PTOX2 affected the hydrogen production in Chlamydomonas.

\section{Discussions}

We established successfully two knock-down mutants, with $90 \%$ and $71 \%$ mRNA reduction of ptox 1 and ptox 2 under $\mathrm{HL}$ stress, respectively. Under normal culture condition, single knock-down of either PTOX did not inhibit cell growth, however, both mutants showed the significantly reduced growth under HL instead, more than $60 \%$ inhibition compared to only $30 \%$ in WT. 
Current modification of PTOX expression, either knock-out or overexpression, shed lights on their multiple cellular functions. Knocking out PTOX in plants or microalgae resulted in severe phenotypes that encompass developmental and growth defects together with increased photosensitivity [36]. Interestingly, down-regulation of PTOX, approximately $3 \%$ of WT levels, did not compromise plant growth, under ambient growth conditions in Arabidopsis [37]. While over-expression of $C$. reinhardtii PTOX1 in plants makes the mutants more sensitive to HL than WT $[38,39]$ and Arabidopsis PTOX in tobacco promotes oxidative stress [40, 41]. Similarly, OsPTOX expression in Synechocystis did not affect growth under standard growth conditions (light intensities between 50 and $150 \mu$ mol photons $\mathrm{m}^{-2} \mathrm{~s}^{-1}$ ) [42]. In other stress treatments, over-expression of PTOX from the salt-tolerant brassica species Eutrema salsugineum show faster induction and a greater final level of PTOX activity once exposed to salt stress [43].

Based on mRNA level comparison between WT and a delta-psbA tobacco plant, up-regulation of the alternative electron transport pathways (NDH complex and PTOX) occurs at the translational or posttranslational levels [44]. This suggests that PTOX is normally in excess, with delicate expression regulations in not only plants but also microalgae.

The differential expression patterns and the complementary relationship of ptoxs under both normal and HL treatments indicate complicate and different potential roles of these two PTOX genes in chlororespiration and stress responses. For instance, the astaxanthin accumulation level was much more in ptox 2 silenced strain than ptox 1 , compared to WT. What's more, significantly increased hydrogen production was observed in ptox 1 silenced strain.

In Glycine max, differential expression of recently duplicated PTOX genes during plant development and stress conditions were extensively investigated [45]. The majority of plant species contain only a single gene encodes PTOX. Previously, two putative PTOX (PTOX1 and 2) genes were identified in Glycine max. In development, PTOX1 was predominant in young tissues, while PTOX2 was more expressed in aged tissues. Under stress conditions, the PTOX transcripts varied according to stress severity, i.e., PTOX1 mRNA was prevalent under mild or moderate stresses while PTOX2 was predominant in drastic stresses. Overall, the results indicate a functional relevance of this gene duplication in G. max metabolism, whereas PTOX1 could be associated with chloroplast effectiveness and PTOX2 to senescence and/or apoptosis [45].

The differential expression of ptoxs was also observed in Chlamydomonas under phosphate deprivation where ptox 2 mRNA level was up-regulated about 13-fold whereas the ptox 1 transcripts increased 2.4-fold after $48 \mathrm{~h}$ of treatment [32]. In a knockout mutant of PTOX2 in Chlamydomonas the plastoquinone pool is constitutively reduced under dark-aerobic conditions, and the ptox 2 mutant shows lower fitness than wild type when grown under phototrophic conditions [33]. In another report, the absence PTOX2 and cytochrome b6f complex of $C$. reinhardtii, results in almost complete reduction of the plastoquinone pool in light [34]. In this study, both ptox genes connected with cell growth inhibition, but with no significantly difference. Interestingly, under normal light intensity, ptox 1 mRNA levels correlated with the growth in both WT and PTOX1i mutant while ptox2 remained relatively low under both WT and PTOX2i strains in 
this study. Thus, ptox 1 may involve in cell growth and ptox 2 plays other metabolic roles in Chlamydomonas.

PTOX is regarded as an enzyme at the crossroads of various metabolic processes, such as regulation of cyclic electron transfer and carotenoid biosynthesis [36]. PTOX is very important for carotenoid biosynthesis, since the phytoene desaturation, a key step in the carotenoid biosynthesis, is blocked in the white sectors of Arabidopsis im mutant [3]. The absence of PTOX in plants usually results in photobleached variegated Arabidopsis leaves [46] and impaired adaptation to environment alteration, and mutant plants will not survive the mediocre light intensity during its early development stage [3]. Although PTOX level and activity has been found to increase under a wide range of stress conditions [9].

Thus, PTOX involves in carotenoid biosynthesis but which ptox gene plays more important role in this process in microalgae is still under investigation.

In other green microalga Haematococcus, Hptox 1 and Hptox 2 also showed differential expression patterns in response to various oxidative stresses [20]. And the authors regarded Hptox 1 as the key PTOX gene for co-regulation of astaxanthin accumulation in Haematococcus [20]. The rice ptox 1 mutant accumulated phytoene in white leaf sectors with a corresponding deficiency in beta-carotene, consistent with the expected function of PTOX1 in promoting phytoene desaturase activity. Our results demonstrate that PTOX1 is required for carotenoid synthesis [47].

Similar to heat and drought, $\mathrm{HL}$ treatment stimulates chlororespiration in higher plants [48] and microalgae [20], causing the up-regulation of the PTOX and the thylakoidal NADH DH complex $[49,50]$. The natural astaxanthin mainly derives from the microalgae producer, Haematococcus. The induction of nitrogen starvation and high light intensity is particularly significant for boosting astaxanthin production [51].

Under HL, ptox 1 mRNA level was up-regulated significantly (more than 10 fold compared to WT) in PTOX2i mutant. Together with our study, it suggests that ptox expressions differ temporally or spatially in response to various stressors. We speculated that it is ptox 1 rather than ptox 2 that is co-regulated, or functionally coupled with carotenoid biosynthesis in Chlamydomonas, similar to Haematococcus.

Hydrogen production in green mciroalgae requires electrons from the photosynthetic electron transfer chain to reduce $\mathrm{H}^{+}$. Sulfur-deprived cultivation of $C$. reinhardtii [52] was previously regarded as the most efficient technique to enhance photobio- $\mathrm{H}_{2}$ production in microalgae [53-55]. Most recently, modification of photosynthetic genes and even non-coding RNAs in Chlamydomonas significantly improve the biohydrog8 en production $[35,56,57]$.

Under $\mathrm{HL}$, ptox 2 responds to the stress with delayed maximum expression level in ptox 1 silenced strainPTOX1i mutant, with a similar but delayed up-regulation level with WT. What's more, significantly increased hydrogen production was observed in the PTOX1i mutant. We address the importance of ptox2 gene in hydrogen bioproduction in Chlamydomonas. 
The limitations of our study are the absence of evidence based on protein levels, and RNAi knock-down still remains relatively high basal expression of the target genes. Further investigations involving in Western blotting and genome editing (for complete individual or double knock-out, if possible) would be highly required for a better understanding of the diverse functions of PTOX genes in microalgae.

\section{Conclusion}

We tried to verify the different functions of two PTOX genes in a model green microalga, by RNAi technique under normal and high light conditions. We propose that PTOX1 may involve in cell growth, cofunctional with astaxanthin biosynthesis while PTOX2 may respond to stress conditions and a better candidate synthetic target for bio-hydrogen bioproduction.

\section{Materials And Methods}

\section{Organism, growth medium, and culture conditions}

Chlamydomonas reinhardtii cell wall-deficient mutant strain CC849 was obtained from the Chlamydomonas Genetic Centre (Duke University, Durham, USA). Microalgal cells were grown using Tris acetate phosphate (TAP) media with mineral nutrient supplements [58] at $25^{\circ} \mathrm{C}$ and under continuous cool-white fluorescent lamps $\left(\approx 100 \mu \mathrm{mol}\right.$ photons $\left./ \mathrm{m}^{2} \mathrm{~s}\right)$.

\section{RNAi vector construction and transformation}

Standard gene cloning methods [59] were used to make the gene constructs. Primers were designed (Table 1) to clone the forward and reserve fragments of ptox 1 (GenBank ID: 5718064), ptox2 (GenBank ID: 5728910$)$ and their introns. The vector for the RNAi-mediated silencing of the ptox 1 and ptox 2 gene was constructed as described previously [60]. Transgenic cells were plated on selective media containing $1.5 \mathrm{mM}$ L-tryptophan, $5 \mu \mathrm{g} / \mathrm{mL}$ paromomycin, and $5 \mu \mathrm{M} 5-\mathrm{Fl}$. We synthesized forward and reverse CDS fragments of ptox 1 and ptox 2 then fused with their introns (Table 1)(Fig. S1) respectively. The products were inserted into plasmid $\mathrm{pH} 124$ for PTOXs silence. All these PCR products with correct length were purified through Takara Agarose-Gel DNA Purification Kit V2.0 and then stored in $-20^{\circ} \mathrm{C}$. The 'hairpin' RNA encoding sequence with plasmid PH124 was constructed by fusing the above available three DNA fragments with SOE-PCR (splicing by overlapping extension-PCR) (Fig. S1). 
Table 1

All primers involved in this study.

\begin{tabular}{|c|c|}
\hline Primer & Sequence \\
\hline ptox1-forward-F & $\begin{array}{l}\text { ctaGCTAGCAGCCGGGCCGCCTACTCGGT } \\
\text { Nhel }\end{array}$ \\
\hline ptox1-forward-R & GAGCTGCACCACCTGCAGGTGTGGGTTGGAAGCGGG \\
\hline ptox1-intron-F & CCCGCTTCCAACCCACACCTGCAGGTGGTGCAGCTC \\
\hline ptox1-intron-R & $\begin{array}{l}\text { cgcGGATCCCTGCAGGTGGTGCAGCTCGT } \\
\text { BamHI }\end{array}$ \\
\hline ptox1-reverse-F & $\begin{array}{l}\text { ctaGGATCCCTGGTGGTGGTGAAGGGAGG } \\
\text { BamHI }\end{array}$ \\
\hline ptox1-reverse-R & $\begin{array}{l}\text { ctaCACGTGAGCCGGGCCGCCTACTCGGT } \\
\text { Pmacl }\end{array}$ \\
\hline ptox2-forward-F & $\begin{array}{l}\text { cgcGCTAGCACGGTGGCGCGCATCCCCTA } \\
\text { Nhel }\end{array}$ \\
\hline ptox2-forward-R & СССТTCСАAAATACACACCTGGCACGCCACCATGGT \\
\hline ptox2-intron-F & ACCATGGTGGCGTGCCAGGTGTGTATTTTGGAAGGG \\
\hline ptox2-intron-R & $\begin{array}{l}\text { ctaGGATCCCTTTGTCGATATGCCGCCCG } \\
\text { BamHI }\end{array}$ \\
\hline ptox2-reverse-F & $\begin{array}{l}\text { cgcGGATCCCTGGCACGCCACCATGGTCT } \\
\text { BamHI }\end{array}$ \\
\hline ptox2-reverse-R & $\begin{array}{l}\text { cgcCACGTGACGGTGGCGCGCATCCCC } \\
\text { Pmacl }\end{array}$ \\
\hline PTOX1-qRT-F & GATGGACACGGAAGCAGCA \\
\hline PTOX1-qRT-R & GTCCGCGAAGTAAACAGGCT \\
\hline PTOX2-qRT-F & GATGGACACGGAAGCAGCA \\
\hline PTOX2-qRT-R & TCTCGAGCACCCAGAACTTCTC \\
\hline$\beta$-Actin-F & ACCCCGTGCTGCTGACTG \\
\hline$\beta$-Actin-R & ACGTTGAAGGTCTCGAACA \\
\hline
\end{tabular}

The pH124-PTOXs-RNAi plasmids were then linearized by Not I and transferred into C. reinhardtii CC849 through the glass bead method [38]. Then qRT-PCR technique was used to screen for the silenced strains with the most significant down-regulation of ptoxs and those with lowest ptoxs levels were selected for following experiments.

\section{DNA and RNA isolations}


We use Universal DNA Extraction Kit Ver.3.0(TAKARA) to isolate genome DNA from $20 \mathrm{~mL}\left(\sim 3 \times 10^{7}\right.$ cells) C. reinhardtii CC849 which were cultured to log phase. RNA from C. reinhardtii CC849 was isolated through FAST200 Kit (FeiJie Shanghai Biotech. Ltd., China).

\section{qRT-PCR}

TaKaRa RNA PCR Kit(AMV) Ver.3.0 (TaKaRa Code DRR019A) and SYBR PrimeScript TM RT-PCR Kit (Perfect Real Time) were used to quantify the transcription level of PTOXs. Specific primers (across intron) for ptoxs and $\beta$-actin were used as the internal reference gene. qRT-PCR analysis was performed on an ABI Prism 7300 Sequence Detection System (Applied Biosystems, USA).

\section{Astaxanthin production under different conditions}

C. reinhardtii CC-849 and transgenic algal strains were cultured in normal light $(\approx 100 \mu \mathrm{mol}$ photons $\left./ \mathrm{m}^{2} \mathrm{~s}\right)$ and high light conditions $\left(\approx 500 \mu \mathrm{mol}\right.$ photons $\left./ \mathrm{m}^{2} \mathrm{~s}\right)$. Astaxanthin content of $C$. reinhardtii cells was analyzed with HPLC according to[20].

\section{Hydrogen production}

C. reinhardtii CC- 849 and transgenic algal strains $(250 \mathrm{~mL})$ were cultured in $500-\mathrm{ml}$ culture bottles sealed with rubber sheet septa until exponential phase in a red light incubator, followed by irradiation with continuous white or blue light to detect hydrogen production. A gas chromatograph was used to detect the concentration of $\mathrm{H}_{2}$ (Agilent 7890A; Agilent Technologies Inc., USA). $\mathrm{H}_{2}, \mathrm{O}_{2}$, and $\mathrm{N}_{2}$ in the gas samples were separated by a molecular sieve column (type $5 \AA$; mesh size 60/80; 6 ft. × 1/8 in. × $2.0 \mathrm{~mm}$ ), and argon was used as the carrier gas. Data were analyzed using $F$ test to test the homogeneity of variance, and then using $t$ test to determine difference significance.

\section{Statistical analysis}

All experiments were repeated at least three times independently, and data were recorded as the mean with standard deviation (SD). Statistical analyses were performed using the Student's $t$ test and Pearson correlation analysis (SPSS13.0). For all of the data analysis, a $p$ value $<0.05$ was considered statistically significant.

\section{Abbreviations}

PTOX: plastid terminal oxidase, PTOX1: plastid terminal oxidase 1, PTOX2: plastid terminal oxidase 2, PTOX1i: PTOX1-RNAi, PTOX2i: PTOX2-RNAi, GH: GHOST, IM: IMMUTANS, RNAi: RNA interference, PSI: photosystem I, PSII: photosystem II, HL: high light, WT: wild type, TAP: Tris acetate phosphate, SOE-PCR: splicing by overlapping extension-PCR.

\section{Declarations}


Ethics Committee of the Shenzhen University approved this study.

\section{Consent to publish}

Not applicable.

\section{Availability of data and materials}

The datasets used and/or analyzed during the current study are available from the corresponding author on reasonable request. All data generated or analyzed during this study are included in this published article.

\section{Competing interests}

The authors declare that the research was conducted in the absence of any commercial or financial relationships that could be construed as a potential conflict of interest. Funding

This work was supported in part by National Natural Science Foundation of China (31670116, 31871734), Guangdong Natural Science Foundation (2018A0303130054), Guangdong Innovation Research Team Fund (2014ZT05S078), and Shenzhen Grant Plan for Science \& Technology (JCYJ20160308095910917, JCYJ20170818100339597, JCYJ20170818143107733) and used for the design of the study, data collection, data analysis, interpretation of data, and in writing the manuscript, respectively.

\section{Authors' contributions}

JXW, ZLH contributed to conception and design of the study. $\mathrm{HL}, \mathrm{CZ}$ and $\mathrm{MX}$ conducted the experiments. $H Q, J C, M J D$ and WLH ran the statistical analysis. HL and CZ wrote the first draft of the manuscript. HL, CZ and JXW wrote the sections of the manuscript. ZLH, APL and JXW contributed to manuscript revision. All authors read and approved the submitted version.

\section{Acknowledgments}

The authors gratefully acknowledge Aaron Wang's careful proofreading and the supports from the Instrumental Analysis Center of Shenzhen University (Xili Campus).

\section{Author details}

Shenzhen Key Laboratory of Marine Bioresources and Eco-environmental Science, Shenzhen Engineering Laboratory for Marine Algal Biotechnology, Guangdong Provincial Key Laboratory for Plant Epigenetics, College of Life Sciences and Oceanography, Shenzhen University, Shenzhen 518060, China.

\section{References}


1. Thiers KLL, da Silva JHM, Sartori GR, Dos Santos CP, Saraiva K, Roque ALM, et al. Polymorphisms in plastoquinol oxidase (PTOX) from Arabidopsis accessions indicate SNP-induced structural variants associated with altitude and rainfall. J Bioenerg Biomembr. 2019;51:151-64.

2. Johnson GN, Stepien P. Plastid terminal oxidase as a route to improving plant stress tolerance: known knowns and known unknowns. Plant Cell Physiol. 2016;57:1387-96.

3. Wang $D$, Fu A. The plastid terminal oxidase is a key factor balancing the redox state of thylakoid membrane. Enzymes. 2016;40:143-71.

4. Aluru MR, Rodermel SR. Control of chloroplast redox by the IMMUTANS terminal oxidase. Physiol Plantarum. 2004;120:4-11.

5. Kuntz M. Plastid terminal oxidase and its biological significance. Planta. 2004;218:896-9.

6. Aluru MR, Yu F, Fu A, Rodermel S. Arabidopsis variegation mutants: new insights into chloroplast biogenesis. J Exp Bo. 2006;57:1871-81.

7. Kambakam S, Bhattacharjee U, Petrich J, Rodermel S. PTOX mediates novel pathways of electron transport in etioplasts of Arabidopsis. Mol Plant. 2016;9:1240-59.

8. Shahbzi M, Gilbert M, Labouré AM, Kuntz M. Dual role of the plastid terminal oxidase in tomato. Plant Physiol. 2007;145:691-702.

9. Sun X, Wen T. Physiological roles of plastid terminal oxidase in plant stress responses. J Biosci. 2011;36:951-6.

10. Díaz M, De haro V, Muñoz R, Quiles MJ. Chlororespiration is involved in the adaptation of Brassica plants to heat and high light intensity. Plant Cell Environ. 2007;30:1578-85.

11. Ibáñez HBA, Muñoz R, Quiles MJ. Chlororespiration and tolerance to drought, heat and high illumination. J Plant Physiol. 2010;167:732-8.

12. Ivanov AG, Rosso D, Savitch LV, stachula P, Rosembert M, Oquist G, et al. Implications of alternative electron sinks in increased resistance of PSIl and PSI photochemistry to high light stress in coldacclimated Arabidopsis thaliana. Photosynth Res. 2012;113:191-206.

13. Segura MV, Quiles MJ. Involvement of chlororespiration in chilling stress in the tropical species Spathiphyllum wallisii. Plant Cell Environ. 2015;38:525-33.

14. Li Q-H, Yao Z-J, Mi H-L. Alleviation of photoinhibition by co-ordination of chlororespiration and cyclic electron flow mediated by NDH under heat stressed condition in Tobacco. Front Plant Sci. 2016;7:285.

15. Hossain MS, Dietz KJ. Tuning of redox regulatory mechanisms, reactive oxygen species and redox homeostasis under salinity stress. Front Plant Sci. 2016;7:548.

16. Tang Y-P, Sun $X$, Wen T, Liu M-J, Yang M-Y, Chen X-F. Implications of terminal oxidase function in regulation of salicylic acid on soybean seedling photosynthetic performance under water stress. Plant Physiol Biochem. 2017;112:19-28.

17. Laureau C, Paepe R, Latouche G, Moreno-Chacón M, Finazzi G, Kuntz M, et al. Plastid terminal oxidase (PTOX) has the potential to act as a safety valve for excess excitation energy in the alpine 
plant species Ranunculus glacialis. L Plant Cell Environ. 2013;36:1296-310.

18. Renato M, Albert B. Joaquín Azcón-Bieto. Respiratory processes in non-photosynthetic plastids. Front Plant Sci. 2015;6:496.

19. Formighieri C, Ceol M, Bonente G, Rochaix JD, Bassi R. Retrograde signaling and photoprotection in a gun4 Mutant of Chlamydomonas reinhardtii. Mol Plant. 2012;5:1242-62.

20. Wang J-X, Sommerfeld M, Hu Q. Occurrence and environmental stress responses of two plastid terminal oxidases in Haematococcus pluvialis (Chlorophyceae). Planta. 2009;230:191-203.

21. Fanciullino AL, Bidel LPR, Urban L. Carotenoid responses to environmental stimuli: integrating redox and carbon controls into a fruit model. Plant Cell Environ. 2014;37:273-89.

22. Bennoun P. The present model for chlororespiration. Photosynthesis Res. 2002;73:273-7.

23. Carol P, Kuntz M. A plastid terminal oxidase comes to light: implications for carotenoid biosynthesis and chlororespiration. Trends Plant Sci. 2001;6:31-6.

24. Simkin AJ, Labouré AM, Kuntz M, Sandmann G. Comparison of carotenoid content, gene expression and enzyme levels in tomato (Lycopersicon esculentum) leaves. Z Naturforsch C. 2003;58:371-80.

25. Josse EM, Simkin AJ, Gaffé J, Labouré AM, Kuntz M, Carol P. A plastid terminal oxidase associated with carotenoid desaturation during chromoplast differentiation. Plant Physiol. 2000;123:1427-36.

26. Jourdan M, Gagné S, Dubois-Laurent $C$, Maghraoui M, Huet S, Suel A, et al. Carotenoid content and root color of cultivated carrot: a candidate-gene association study using an original broad unstructured population. PLoS One. 2015;10:e0116674. doi:.

27. Barr J, White WS, Chen L, Bae H, Rodermel S. The GHOST terminal oxidase regulates developmental programming in tomato fruit. Plant Cell Environ. 2004;27:840-52.

28. Cynthia RF, Ross BN, Martens GD. An antibody against a conserved C-terminal consensus motif from plant alternative oxidase (AOX) isoforms 1 and 2 label plastids in the explosive dwarf mistletoe (Arceuthobium americanum, Santalaceae) fruit exocarp. Protoplasma. 2013;250:317-23.

29. Hao Z, Zong Y, Liu H, Tu Z, Li H. Cloning, characterization and functional analysis of the LtuPTOX gene, a homologue of arabidopsis thaliana IMMUTANS derived from Liriodendron tulipifera. Genes (Basel). 2019;10.

30. Gwak Y, Hwang YS, Wang BB, Kim M, Jeong J, Lee CG, et al. Comparative analyses of lipidomes and transcriptomes reveal a concerted action of multiple defensive systems against photooxidative stress in Haematococcus pluvialis. J Exp Bot. 2014;65:4317-34.

31. Zhang L-T, Su F, Zhang C-H, Gong F-Y, Liu J-G. Changes of photosynthetic behaviors and photoprotection during cell transformation and astaxanthin accumulation in Haematococcus pluvialis grown outdoors in tubular photobioreactors. Int J Mol Sci. 2016;18:33.

32. Moseley JL, Chang C-W, Grossman AR. Genome-based approaches to understanding phosphorus deprivation responses and PSR1 control in Chlamydomonas reinhardtii. Eukaryot Cell. 2006;5:26-44.

33. Houille-Vernes L, Rappaport F, Wollman FA, Alric J, Johnson X. Plastid terminal oxidase 2 (PTOX2) is the major oxidase involved in chlororespiration in Chlamydomonas. Proc Natl Acad Sci USA. 
2011;108:20820-5.

34. Brzezowski P, Ksas B, Havaux M, Grimm B, Chazaux M, Peltier G, et al. The function of PROTOPORPHYRINOGEN IX OXIDASE in chlorophyll biosynthesis requires oxidised plastoquinone in Chlamydomonas reinhardtii. Commun Biol. 2019;2:159.

35. Wang Y, Zhuang X, Chen M, Zeng Z, Cai X, Li H, et al. An endogenous microRNA (miRNA1166.1) can regulate photobio- $\mathrm{H}_{2}$ production in eukaryotic green alga Chlamydomonas reinhardtii. Biotechnol Biofuels. 2018;11:126.

36. Nawrocki WJ, Tourasse NJ, Taly A, Rappaport F, Wollman FA. The plastid terminal oxidase: its elusive function points to multiple contributions to plastid physiology. Annu Rev Plant Biol. 2015;66:49-74.

37. Fu A, Aluru M, Rodermel SR. Conserved active site sequences in Arabidopsis plastid terminal oxidase (PTOX): in vitro and in planta mutagenesis studies. J Biol Chem. 2009;284:22625-32.

38. Ahmad N, Michoux F, Nixon PJ. Investigating the production of foreign membrane proteins in tobacco chloroplasts: expression of an algal plastid terminal oxidase. PLoS One. 2012;7:e41722. .

39. Feilke K, Streb P, Cornic G, Perreau F, Kruk J, Krieger-Liszkay A. Effect of Chlamydomonas plastid terminal oxidase 1 expressed in tobacco on photosynthetic electron transfer. Plant J. 2016;85:21928.

40. Joët T, Genty B, Josse E-M, Kuntz M, Cournac L, Peltier G. nvolvement of a plastid terminal oxidase in plastoquinone oxidation as evidenced by expression of the Arabidopsis thaliana enzyme in tobacco. J Biol Chem. 2002;277:31623-30.

41. Heyno E, Gross CM, Laureau C, Culcasi M, Pietri S, Krieger-Liszkay A. Plastid alternative oxidase (PTOX) promotes oxidative stress when overexpressed in tobacc. J Biol Chem. 2009;284:31174-80.

42. Feilke K, Ajlani G, Krieger-Liszkay A. Overexpression of plastid terminal oxidase in Synechocystis sp. PCC 6803 alters cellular redox state. Philos Trans R Soc Lond B Biol Sci. 2017;372. doi:.

43. Stepien P, JohnsonG N. Plastid terminal oxidase requires translocation to the grana stacks to act as a sink for electron transport. Proc Natl Acad Sci USA. 2018;115:9634-9.

44. Baena-González E, Allahverdiyeva Y, Svab Z, Maliga P, Josse EM, Kuntz M, et al. Deletion of the tobacco plastid psbA gene triggers an upregulation of the thylakoid-associated $N A D(P) H$ dehydrogenase complex and the plastid terminal oxidase (PTOX). Plant J. 2003;35:704-16.

45. Maia RA, da Cruz Saraiva KD, Roque ALM, Thiers KLL, Dos Santos CP, da Silva JHM, et al. Differential expression of recently duplicated PTOX genes in Glycine max during plant development and stress conditions. J Bioenerg Biomembr. 2019;51:355-70.

46. Foudree A, Putarjunan A, Kambakam S, Nolan T, Fussell J, Pogorelko G, et al. The mechanism of variegation in immutans provides insight into chloroplast biogenesis. Front Plant Sci. 2012;3:1-10.

47. Tamiru M, Abe A, Utsushi H, Yoshida K, Takagi H, Fujisaki K, et al. The tillering phenotype of the rice plastid terminal oxidase (PTOX) loss-of-function mutant is associated with strigolactone deficiency. New Phytol. 2014;202:116-31. 
48. Quiles MJ. Stimulation of chlororespiration by heat and high light intensity in oat plants. Plant Cell Environ. 2006;29:1463-70.

49. Tallon C, Quiles MJ. Acclimation to heat and high light intensity during the development of oat leaves increases the NADH DH complex and PTOX levels in chloroplasts. Plant Sci. 2007;173:43845.

50. Soto A, Hernandez L, Quiles MJ. High root temperature affects the tolerance to high light intensity in Spathiphyllum plants. Plant Sci. 2014;227:84-9.

51. Zhang W-W, Zhou X-F, Zhang Y-L, Cheng P-F, Ma R, Cheng WL, et al. Enhancing astaxanthin accumulation in haematococcus pluvialis by coupled light intensity and nitrogen starvation in column photobioreactors. J Microbiol Biotechnol. 2018;28:2019-28.

52. Melis A, Zhang L, Forestier M, Ghirardi ML, Seibert M. Sustained photobiological hydrogen gas production upon reversible inactivation of oxygen evolution in the green alga Chlamydomonas reinhardtii. Plant Physiol. 2000;122:127-36.

53. Hemschemeier A, Fouchard S, Cournac L, Peltier G, Happe T. Hydrogen production by Chlamydomonas reinhardtii: an elaborate interplay of electron sources and sinks. Planta. 2008;227:397-407.

54. Melis A, Happe T. Trails of green alga $\mathrm{H}_{2}$-production research-from Hans Gaffron to new frontiers. Photosyn Res. 2004;80:401-9.

55. Zhang L, Happe T, Melis A. Biochemical and morphological characterization of sulfur-deprived and $\mathrm{H}_{2}$-producing Chlamydomonas reinhardtii (green alga). Planta. 2002;214:552-61.

56. Li H, Liu Y-M, Wang Y-T, Chen M-C, Zhuang X-S, Wang C-G, Wang J-X, et al. Improved photobio- $\mathrm{H}_{2}$ production regulated by artificial miRNA targeting psbA in green microalga Chlamydomonas reinhardtii. Biotechnol Biofuels. 2018;11:36.

57. Wang Y-T, Jiang X-H, Hu C-X, Sun T, Zeng Z-Y, Cai X-Q, et al. Optogenetic regulation of artificial microRNA improves $\mathrm{H} 2$ production in green alga Chlamydomonas reinhardtii. Biotechnol Biofuels. 2017; 10:257.

58. Gorman DS, Levine RP. Cytochrome $f$ and plastocyanin: their sequence in the photosynthetic electron transport chain of Chlamydomonas reinhardti. P Natl Acad Sci USA. 1965;54:1665-9.

59. Sambrook J, Fritsch EF, Maniatis T. Molecular Cloning: A Laboratory Manual. 2nd ed. New York: CSHL Press; 1989.

60. Luo Q-L, Song W-W, Li Y-J, Wang C-G, Hu Z-L. Flagella-Associated WDR-Containing Protein CrFAP89 Regulates Growth and Lipid Accumulation in Chlamydomonas reinhardtii. Front Plant Sci. 2018;9:691.

\section{Figures}




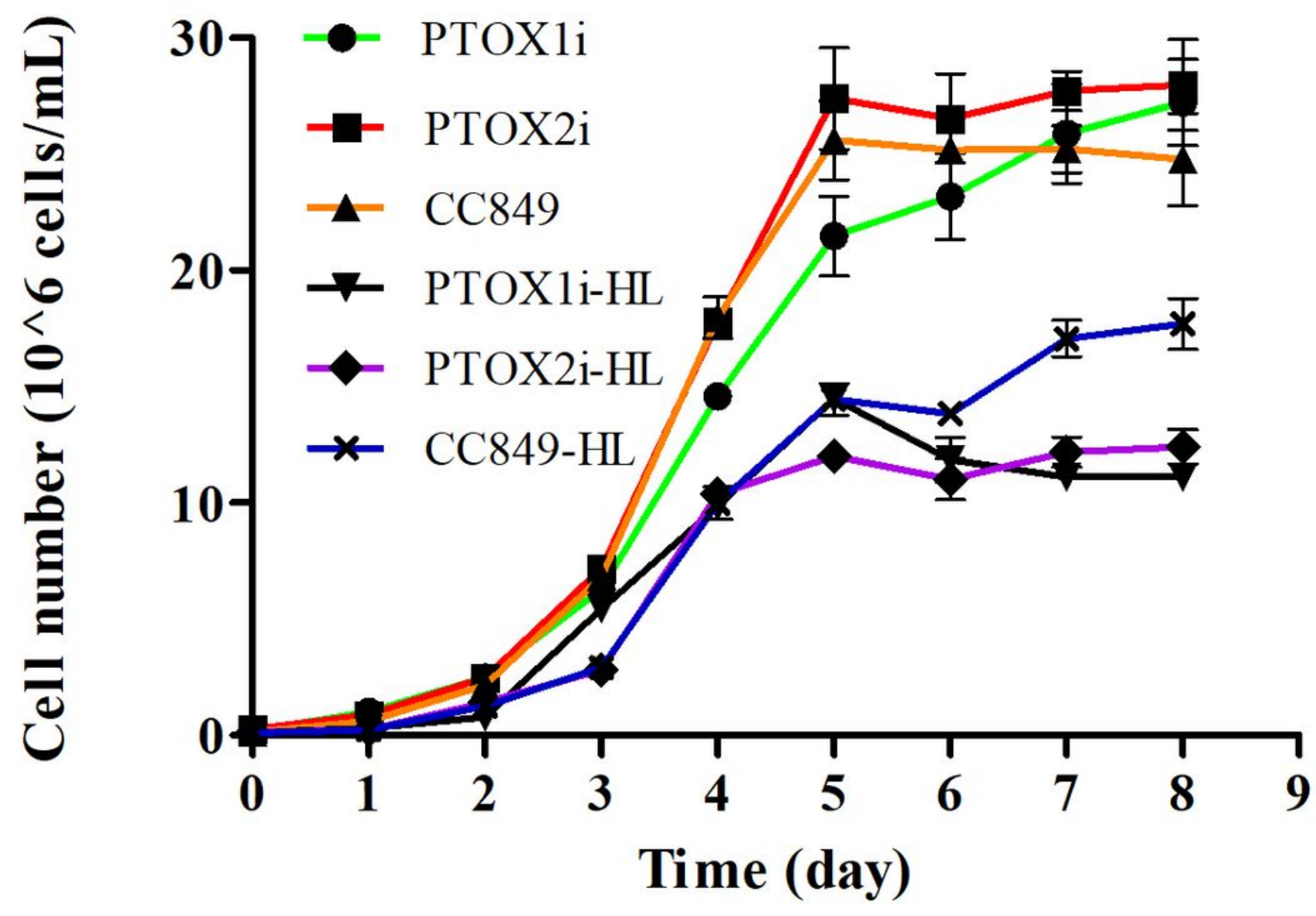

Figure 1

Growth curves of WT (CC849) and PTOX mutants (PTOX1i as ptox1 interfered, PTOX2i as ptox2 interfered), under normal light density and high light culture condition $(-\mathrm{HL})$. Cell numbers are shown as $10^{\wedge} 6$ cells $/ \mathrm{mL}$. 
Fig. 2a

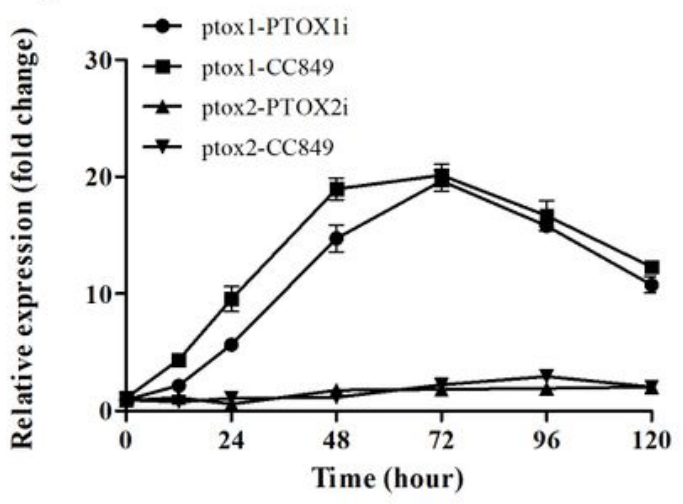

Fig. 2b

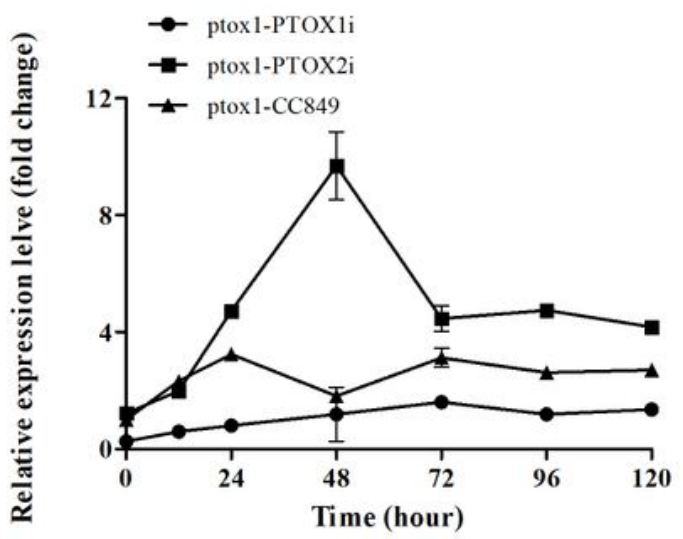

Fig. 2c

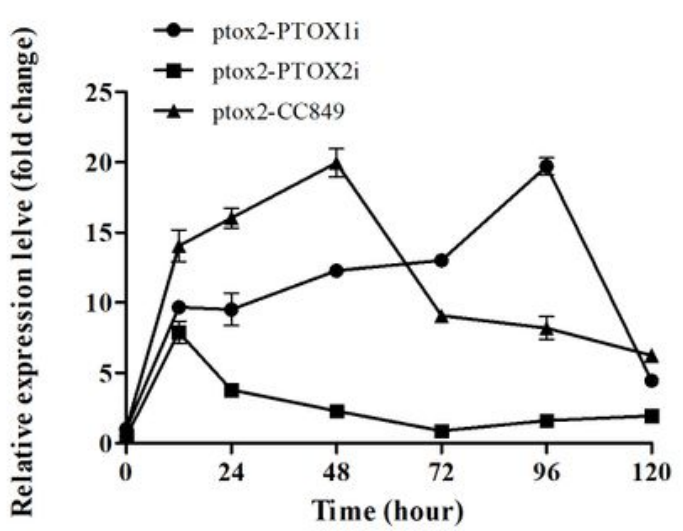

\section{Figure 2}

Relative expression levels (fold change) of ptox 1,2 genes in WT and their respective mutants under normal light density (Fig 2a), ptox1 in WT two mutants under HL culture condition (Fig 2b) and ptox2 in WT and mutants under HL culture condition (Fig 2c). 


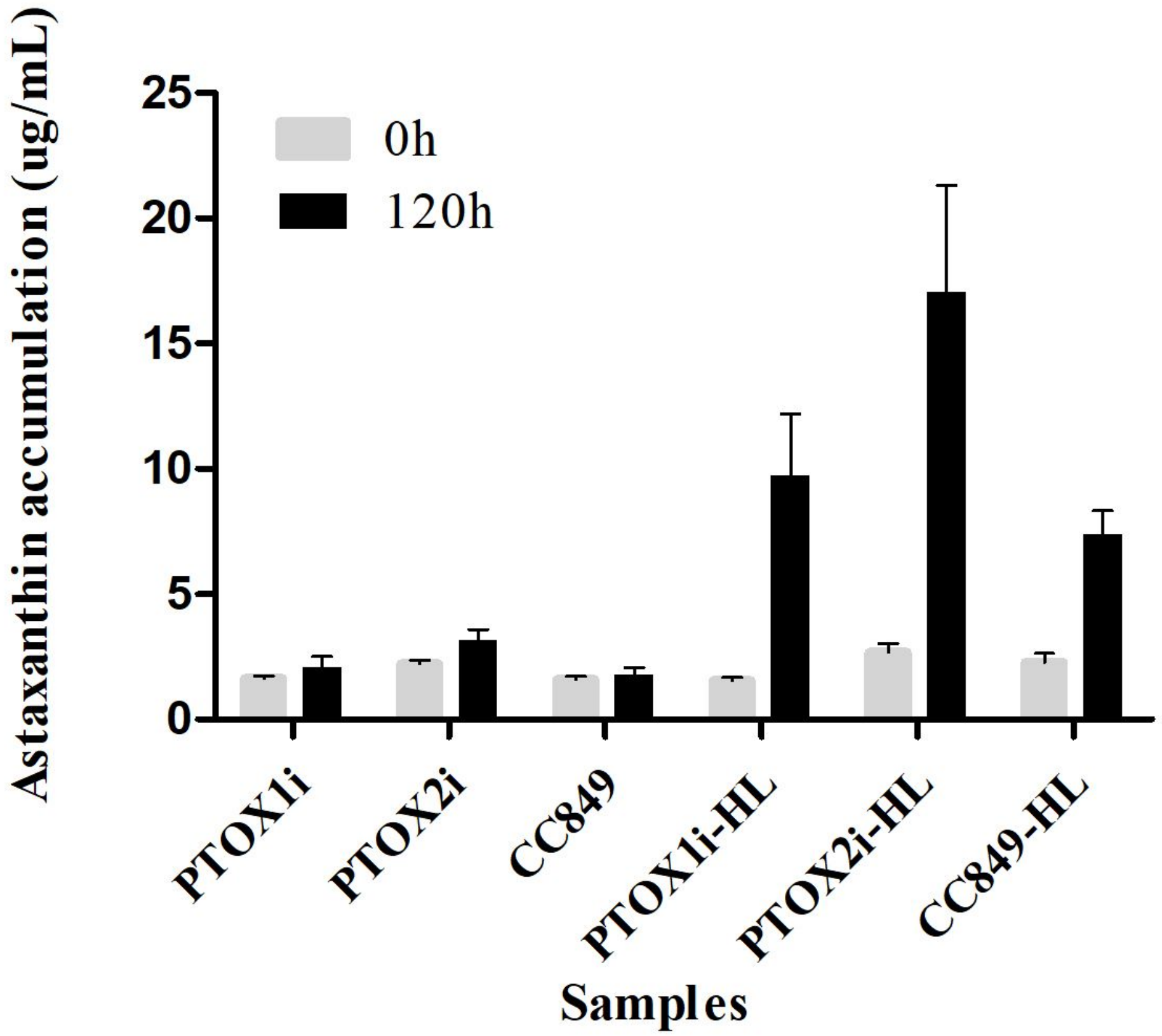

Figure 3

Astaxanthin accumulation $(\mu \mathrm{g} / \mathrm{mL})$ in WT and mutants (PTOX1i as ptox1 interfered, PTOX2i as ptox2 interfered) under normal light density and HL culture conditions. 


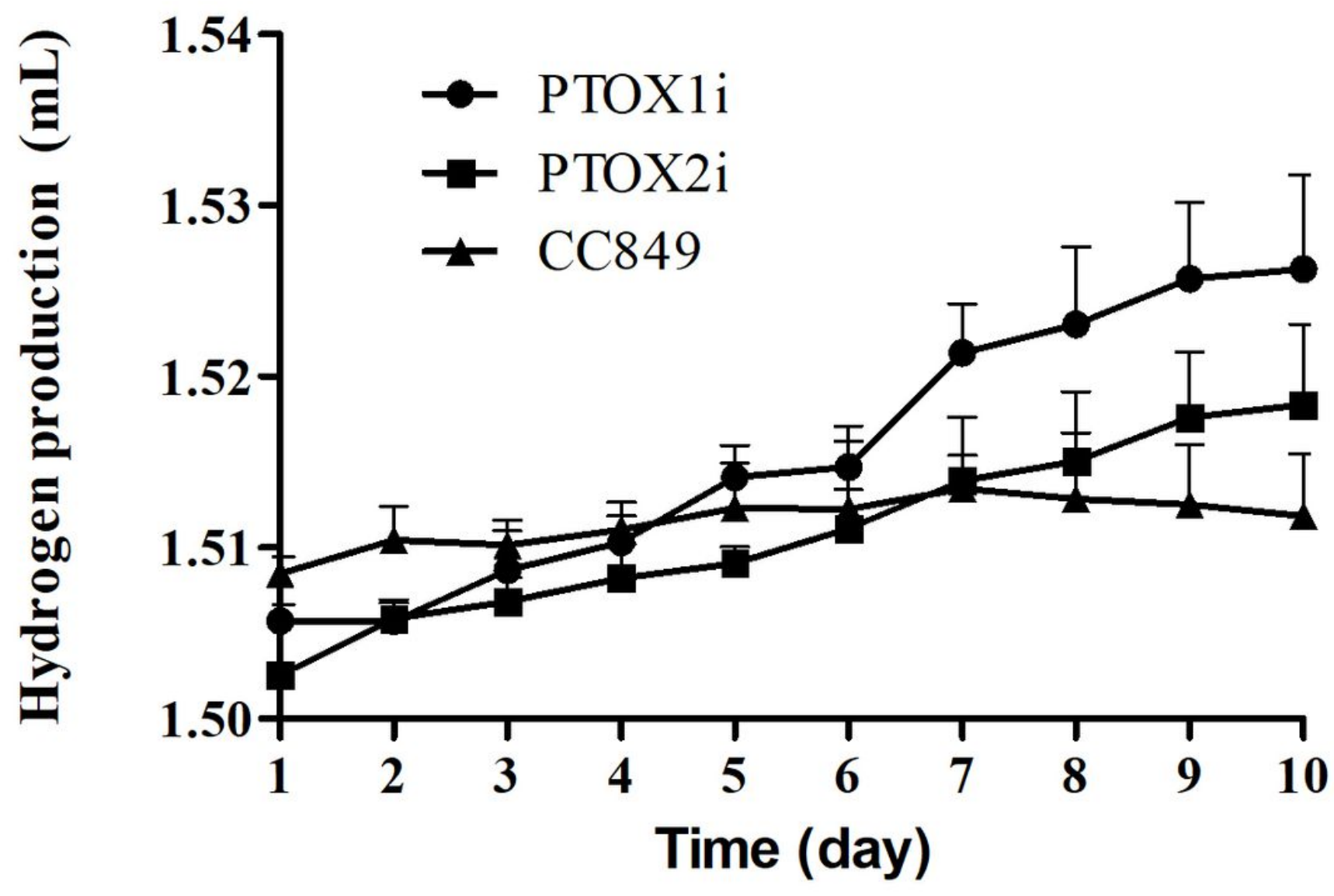

Figure 4

Biohydrogen production $(\mathrm{mL})$ per day in WT and mutants (PTOX1i as ptox1 interfered, PTOX2i as ptox2 interfered) under normal light density condition.

\section{Supplementary Files}

This is a list of supplementary files associated with this preprint. Click to download.

- Fig.S3.png

- Fig.S2.png

- Fig.S1.jpg 